\title{
The investigation of the relation between expansion strategy and outcomes of two-stage expander-implant breast reconstruction
}

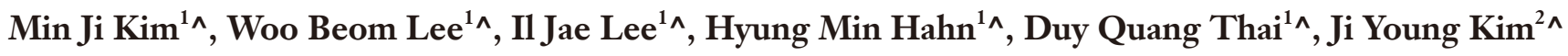 \\ ${ }^{1}$ Department of Plastic \& Reconstructive Surgery, Ajou University School of Medicine, Suwon, Korea; ${ }^{2}$ Department of Surgery, Ajou University \\ School of Medicine, Suwon, Korea \\ Contributions: (I) Conception and design: MJ Kim, WB Lee, IJ Lee; (II) Administrative support: HM Hahn, IJ Lee; (III) Provision of study materials \\ or patients: WB Lee, DQ Thai; (IV) Collection and assembly of data: MJ Kim, WB Lee, DQ Thai; (V) Data analysis and interpretation: MJ Kim, \\ WB Lee, JY Kim; (VI) Manuscript writing: All authors; (VII) Final approval of manuscript: All authors \\ Correspondence to: Ji Young Kim, MD. Clinical Associate Professor, Department of Surgery, Ajou University School of Medicine, 164 World Cup-ro, \\ Yeongtong-gu, Suwon 16499, Korea. Email: gsjiyoung@gmail.com.
}

Background Numerous risk factors for the complications of two-stage, immediate implant-based breast
reconstruction have been identified, although few studies have directly examined the impact of breast size
and expansion protocols on the surgical outcomes of breast reconstruction. This study aimed to evaluate
the impact of breast size, expansion velocity, and volume-related variables on postoperative complications of
breast reconstruction.

Methods: The cohort involved patients who underwent immediate breast expander reconstruction at a single center between 2017 and 2019. The breast size was classified into three categories according to the weight of the mastectomy specimen as small (<300 g), medium ( $\geq 300 \mathrm{~g}, \leq 500 \mathrm{~g})$, or large $(>500 \mathrm{~g})$. Multifactorial logistic regressions were used to assess the impact of variables, and receiver operating characteristic (ROC) curve analysis was used to determine the optimal cut-off value for predicting the complication event.

Results: Of the 174 breasts (168 patients), 51 (29.3\%), 66 (37.9\%), and 57 (32.6\%) breasts were classified as small, medium, and large, respectively. The rate of infection $(\mathrm{P}=0.014)$ and expander/implant failure $(\mathrm{P}=0.007)$ significantly differed according to breast size, with the rate being the highest in large breasts. Multivariate logistic regression analysis showed that body mass index (BMI) [odds ratio (OR): 1.25; $\mathrm{P}=0.003$ ], nipple-sparing mastectomy (OR: 2.82; $\mathrm{P}=0.036$ ), sentinel biopsy ( $\mathrm{OR}: 5.10 ; \mathrm{P}=0.016$ ), final expansion volume (OR: 0.99; $\mathrm{P}=0.022)$, and expansion velocity (OR: 0.703; $\mathrm{P}=0.024)$ were significant independent predictors of any complication. In the ROC analysis, breast weight $>696 \mathrm{~g}$ could predict the possibility of revision surgery, with a sensitivity of $42.9 \%$ and specificity of $81.8 \%$.

Conclusions: The final expansion volume and expansion velocity have a significant negative relationship with overall complications in breast reconstruction. A standard expansion protocol needs to be established to ensure the success of two-stage breast reconstruction.

Keywords: Breast cancer; mammaplasty; complications; breast implantation; tissue expansion devices

Submitted Aug 02, 2021. Accepted for publication Nov 05, 2021.

doi: $10.21037 /$ gs-21-515

View this article at: https://dx.doi.org/10.21037/gs-21-515

^ ORCID: Min Ji Kim, 0000-0002-0723-4382; Woo Beom Lee, 0000-0002-5333-5424; Il Jae Lee, 0000-0002-9478-6969; Hyung Min Hahn, 0000-0002-4756-1804; Duy Quang Thai, 0000-0002-7216-2514; Ji Young Kim, 0000-0001-5539-7269. 


\section{Introduction}

The increasing rate of breast cancer has also increased the number of postmastectomy breast reconstructions performed. In the past, breast reconstruction was performed as a delayed procedure largely because of concerns that the reconstructive process would compromise adjuvant oncologic treatment such as chemo-or radiotherapy (1). However, current strategies of surgical breast reconstruction can achieve both satisfactory therapeutic and cosmetic results (2). Implant-based breast reconstruction is associated with superior aesthetic benefits and rapid patient recovery. However, its use is limited in patients indicated for postmastectomy radiation therapy. These patients have weak connective tissues and inadequate soft-tissue coverage, leading to skin breakdown, implant exposure, and suboptimal aesthetic outcomes with firm, highriding breasts with poor symmetry $(3,4)$. Tissue response to radiation exposure varies temporally (5), and the most common cause of breast reconstruction failure is longterm radiation-induced tissue injury. Thus, a two-stage breast reconstruction technique that has the advantages of minimizing the radiation effect and maximizing implant aesthetics has been introduced. The two-stage implantbased reconstruction performed immediately after mastectomy is currently the preferred method owing to its advantages of improved aesthetic results, equivalent oncologic outcomes, and decreased costs. It is also the optimal strategy for breast surgery with autologous breast reconstruction (6).

Several factors influence the outcomes of two-stage breast reconstruction. Smoking, obesity, and the reconstructive method have been reported to associate with the risk of complications in implant-based reconstruction (7). In addition, the size of the breast remains a challenge in expander reconstruction (8). A large breast size yields a larger surface area for the mastectomy flap, increasing the possibility of impaired perfusion and ischemic sequelae with subsequent reconstruction. Another influencing factor of the surgical outcomes of two-stage breast reconstruction is the expansion protocol. Although several trials have supported the usefulness of reconstructive surgery, clinical evidence on the expansion protocol is still limited. Initial saline administration immediately after expander insertion increases its final volume to up to $20-30 \%$, while other studies have reported complete deflation (9). The completion of chemotherapy and/or radiotherapy also varies. Further, the expander inflation interval and maximal inflated volume can also differ.

Further, patient care differs between immediate and delayed breast reconstruction, and the expansion protocol, including inflation, deflation volume, and expansion velocity, differs by patient. However, few studies have directly examined the impact of breast size and expansion protocols on the surgical outcomes of breast reconstruction. Thus, the purpose of our study was to evaluate the effect of expansion protocol, including breast size and expansion velocity, on the risk of complications in two-stage breast reconstruction surgery to elucidate the possible causes of reconstruction failure. To this end, the breast was classified by size and the rate of complications was compared among groups. The risk factors of surgical complication were also investigated.

We present the following article in accordance with the STARD reporting checklist (available at https:// gs.amegroups.com/article/view/10.21037/gs-21-515/rc).

\section{Methods}

\section{Study design and patients}

This retrospective study evaluated patients who underwent immediate breast expander reconstruction at a single center (Ajou University Hospital, Suwon, Korea) between 2017 and 2019. We collected the patents' data from electronic medical records (EMR) system of Ajou University Hospital. Patients who underwent two-stage prosthetic breast reconstruction (tissue expander followed by permanent implant) following modified radical mastectomy. The study inclusion criteria was postoperative follow up for a minimum of 6 months, and the follow up range was 11.3-37.1 months. Exclusion criteria for this study was included the patients who received the one staged breast reconstruction (e.g., direct to implant insertion or autologous reconstruction) or autologous reconstruction after operation of tissue expander and whose follow-up period was less than 6 months. Age, body mass index (BMI), cancer profile and stage, volume of mastectomy specimen, initial and final expander volume, history of radiation therapy and/or chemotherapy, history of diabetes and other comorbidities, history of smoking, and the occurrence of complication were recorded for each patient. For patients who underwent bilateral breast reconstruction, data from each breast were recorded as an independent point.

Given that breast size is the primary consideration in expander-based breast reconstruction, breast size was 
stratified into three categories according to the weight of the specimen for total mastectomy: small breast, specimen weight $<300 \mathrm{~g}$ (10); medium, 300-500 g; and large, $>500 \mathrm{~g}(11,12)$. The study was conducted in accordance with the Declaration of Helsinki (as revised in 2013) and was approved by the Institutional Review Board of Ajou University Hospital (approval number: MEDMDB-21-235). The need for informed consent was waived owing to the retrospective nature of the research. However, consent to obtain and to publish relevant images was obtained from all participants. The contents were included in the consent form for surgery.

\section{Reconstruction technique}

All patients underwent immediate breast reconstruction following modified radical mastectomy. The device was partly placed into a space created beneath the pectoralis major muscle supero-medially, and the expander was inserted using the prepectoral plane. In all patients, acellular dermal matrix (CG-cryoderm ${ }^{\circledR}$, CGBio Corp., Seongnam, Korea) was used to cover the infero-lateral surface of the expander. The size of the acellular dermal matrix ranged from 60 to $324 \mathrm{~cm}^{2}$. Complete coverage with muscular and acellular dermal matrix was considered to protect the tissue expander and to help conceal the flaws of the permanent implant. In only one patient, the prepectoral plane was used for the implant.

In the case of insertion at the prepectoral plane, the whole surface of the expander was covered with the acellular dermal matrix. After placement of the expander and completion of suturing, normal-saline with indigo carmine was injected into the expander for initial inflation, depending on the condition of patient's mastectomy flap. Usually, an expander of the same size as the mastectomy volume was chosen. However, if contralateral augmentation was planned, mastectomy dissection area was wider than expected, or axillary lymph node dissection resulted in lateral dimpling, the physician would prefer to insert a larger expander. Initial inflation volume was decided by skin durability, and it was usually lesser than $1 / 3^{\text {rd }}$ of the expander size. This completed the first surgery. Expander fillings were usually initiated 2-4 weeks after surgery and were performed using chlorhexidine preparation of the port site. We performed biweekly patient follow up with expander filling of 50-100 cc/14 days; however, expansion protocol varied depending on the status of patient's skin flap and patient's compliance. Patients receiving adjuvant chemotherapy underwent expansion throughout the duration of chemotherapy. Patients requiring adjuvant radiation therapy underwent rapid expansion followed by radiation therapy (13).

The second stage of reconstruction comprised removal of the tissue expander and a capsulotomy or capsulectomy, followed by the placement of a permanent silicone gel and autologous fat graft for supero-medial side depression, if necessary. Radiotherapy and chemotherapy were performed either before the first surgery or after completion of the second surgery (Figure 1). For patients with a history of radiation therapy, the second stage of reconstruction was delayed until at least 6 months from the completion of the radiation therapy to allow full healing of the wound from radiation injury (Figure 2).

\section{Study variables}

The factors of the expansion protocols were then evaluated (Figure 3). The mastectomy volume (cc) and weight (g) were recorded after mastectomy by GS-team. In the stage of expander insertion operation, intraoperatively, we made a note regarding the extent of initial expander inflation. The serial skin expansion from the first to the second surgery, expansion volume and its interval, and final inflation volume (cc) were also recorded. The accumulated inflation volume after serial follow up was added to the initial inflation volume to calculate the final inflation volume. In our protocol, the final expansion volume limit was never over $120 \%$ of the designated expander volume. The final implant volume and postoperative follow up status were determined. Expansion velocity was calculated by subtracting the initial inflation volume from the final inflation volume and dividing this by the inflation period as follows:

Expansion velocity $\left(\frac{c c}{\text { day }}\right)=\frac{\text { Final inflation volume }(c c)-\text { Initial inflation volume }(c c)}{\text { Inflation period }: 2 \text { nd operation date } \sim 1 \text { st inflation date }(\text { days })}$

\section{Assessments}

Complications of infection, seroma and/or hematoma, skin necrosis including nipple, and wound dehiscence were recorded. Complications were assessed from immediately after the first expander insertion surgery to follow up after completion of second tissue expander and implant change operation. If a complication was noted at any point of time, the event of that complication was recorded. Infections were defined as surgical events that needed additional intravenous antibiotics or ex-plantation at least $2-3$ weeks after 

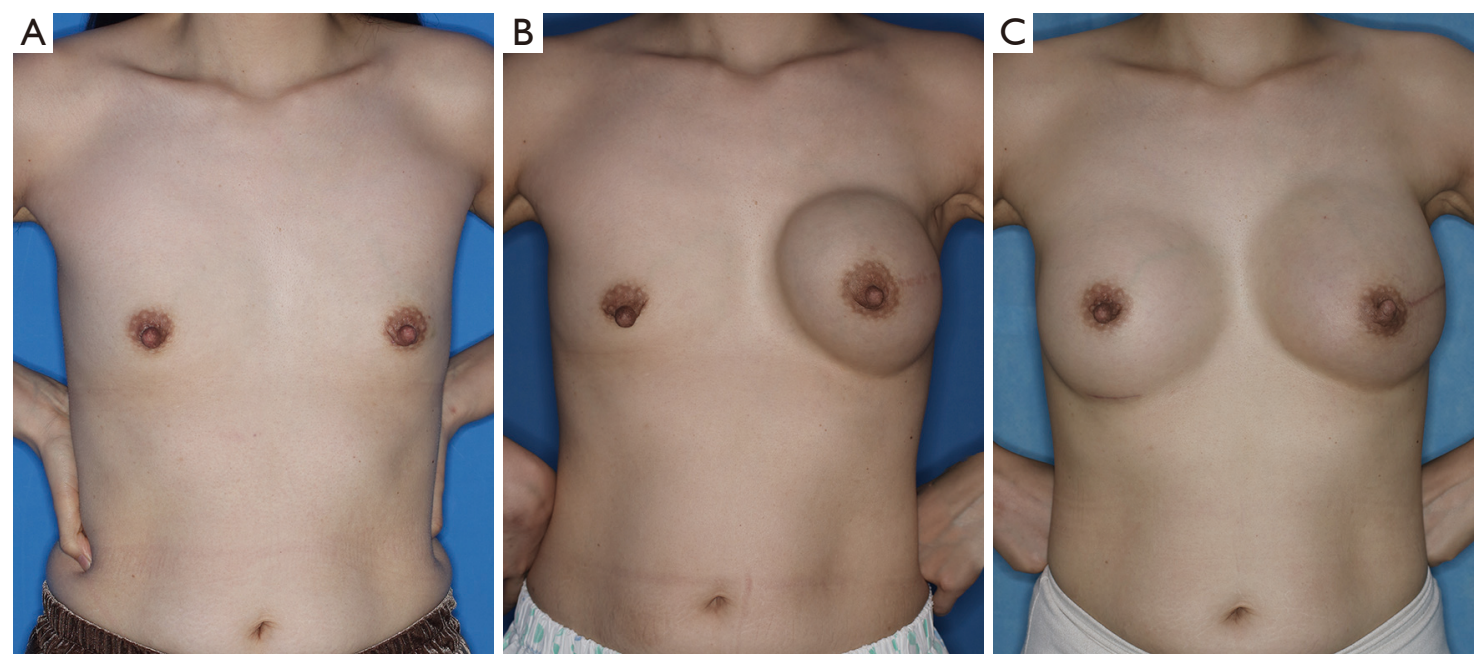

Figure 1 Representative case of two-stage expander-implant breast reconstruction following nipple-sparing mastectomy. A 38-year-old woman with left breast cancer, invasive ductal carcinoma, and a history of adjuvant chemotherapy underwent unilateral nipple-sparing mastectomy followed by immediate reconstruction with subpectoral insertion of a $350 \mathrm{cc}$ tissue expander. The weight and volume of the mastectomy specimen were $240 \mathrm{~g}$ and $300 \mathrm{cc}$, respectively, and the initial fill volume was $130 \mathrm{cc}$. The final fill volume was $360 \mathrm{cc}$, and the expansion velocity was $230 \mathrm{cc} / 58$ days. At 5 months after the expander surgery, she underwent a second surgery for expander/implant exchange with contralateral augmentation by silicone implant insertion. (A) Preoperative views; (B) full expanded state; (C) 1 year after surgery.
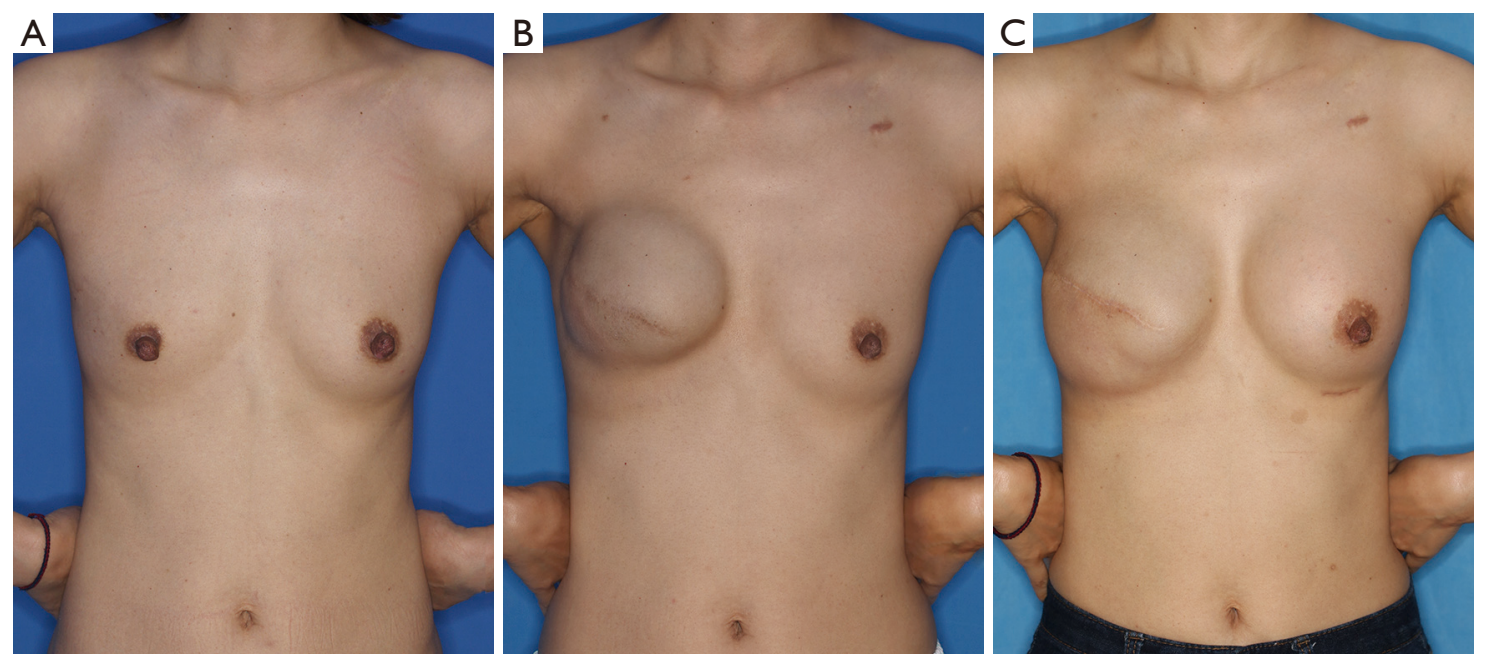

Figure 2 Representative case of two-stage expander-implant breast reconstruction following skin-sparing mastectomy. A 43-year-old woman with right breast cancer, invasive lobular carcinoma, and a history of adjuvant chemotherapy and radiotherapy underwent unilateral skinsparing mastectomy followed by immediate reconstruction with subpectoral insertion of a $350 \mathrm{cc}$ tissue expander. The weight and volume of the mastectomy specimen were $280 \mathrm{~g}$ and $320 \mathrm{cc}$, respectively, and the initial fill volume was $100 \mathrm{cc}$. The final fill volume was $350 \mathrm{cc}$, and the expansion velocity was $250 \mathrm{cc} / 94$ days. At 20 months after the expander surgery (12 months after the completion of radiotherapy), she underwent a second surgery for expander/implant exchange with contralateral augmentation by silicone implant insertion. (A) Preoperative views; (B) full expanded state; (C) 1 year after surgery. 
$1^{\text {st }}$ OPERATION

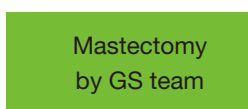
by GS team
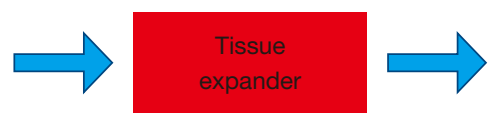

Serial skin expansion $2^{\text {nd }}$ OPERATION

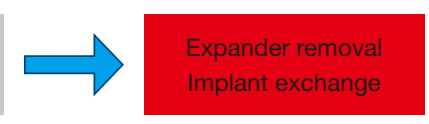

Inflation period
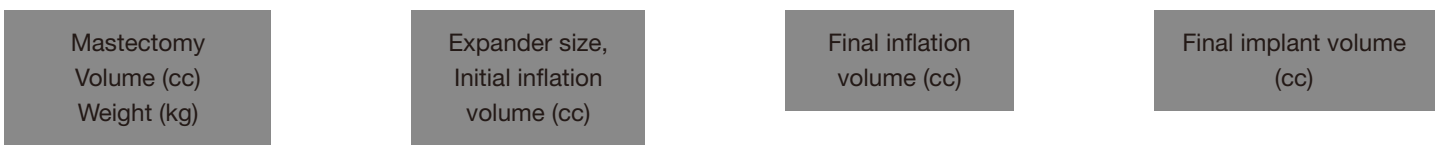

Figure 3 Schematic diagram of the expansion protocol variables in two-stage breast reconstruction.

surgery expander/implant failure was defined as any type of infection-related device failure, including spontaneous rupture or inadvertent iatrogenic puncture, displacement of the device requiring revision, painful capsular contracture, and exchange for another prosthesis. Revision surgery was defined as a minor surgical procedure (e.g., debridement or repair) for any complication.

\section{Statistical analyses}

Continuous variables were presented as the means and standard deviations (SDs) and were compared using the $t$-test. Meanwhile, categorical variables were presented as frequencies and percentages and were compared using the chi-square and Fisher's exact tests. The risk factors for overall complication were first identified through a univariate analysis, and significant variables (i.e., those with a $\mathrm{P}$ value $<0.05$ ) were included in the multivariate analysis. Backward-stepwise logistic regression analysis was performed to identify risk factors for surgical complication. Receiver operating characteristic (ROC) curve analysis was used to determine the optimal cut-off value predictive of the complication event. All statistical analyses were performed using SPSS Version 19.0 (IBM Corp., Armonk, NY, USA). A $P$ value of $<0.05$ was considered statistically significant.

\section{Results}

\section{Patient and breast characteristics}

A total of 168 patients and 174 breasts with a mean age of 43.39 years (range, $24-75$ years) were evaluated. The mean BMI was $22.37 \mathrm{~kg} / \mathrm{m}^{2}$ (range, $15.7-31.2 \mathrm{~kg} / \mathrm{m}^{2}$ ). The most prevalent cancer type was invasive ductal cell carcinoma
(52.9\%), followed by ductal carcinoma in situ (33.9\%). Overall, $18.4 \%$ of the patients had comorbid conditions [i.e., controlled diabetes, hypertension (HTN), and thyroid disease], and only 3 patients were current smokers. Of the 174 breasts (168 patients), 51 (29.3\%), 66 (37.9\%), and 57 (32.6\%) breasts were classified as small, medium, and large, respectively. The mean weight and volume for small breasts were $217.43 \mathrm{~kg}$ and $232.54 \mathrm{cc}$; medium breasts, $399.43 \mathrm{~kg}$ and $427.65 \mathrm{cc}$; and large breasts, $693.89 \mathrm{~kg}$ and $748.15 \mathrm{cc}$, respectively. Obesity (BMI $>30 \mathrm{~kg} / \mathrm{m}^{2}$ ) was observed in patients with large breasts $(15.69 \%)$ alone. The most common type of mastectomy for medium and large breasts was nipple-sparing mastectomy $(60.6 \%$ in medium group and $54.4 \%$ in large group respectively), whereas it was skinsparing mastectomy in small breasts (39.2\%). Neoadjuvant radiotherapy was more commonly performed in small and medium breasts, while adjuvant radiotherapy was more frequent in large breasts. Chemotherapy was primarily performed in the neoadjuvant setting in small $(23.52 \%)$ and large breasts (31.58\%), respectively (Table 1).

\section{Complications}

Complications during the reconstruction period occurred in 72 breasts, with the most common being skin necrosis $(24.7 \%)$. Other complications included surgical site infection $(3.4 \%)$, seroma or hematoma $(11.5 \%)$, revision surgery $(9.7 \%)$, and expander/implant failure (4.0\%). The rate of any complications was $41.3 \%$ (Table 2). The rates of surgical site infection $(\mathrm{P}=0.014)$ and expander/implant failure $(\mathrm{P}=0.007)$ significantly differed according to breast size, with the rates being the highest in large breasts. Meanwhile, skin necrosis was significantly more frequent in medium breasts $(\mathrm{P}=0.026)$. The rates of seroma/hematoma 
Table 1 Patient demographics and mastectomy-related variables stratified by size of breast determined by weight of mastectomy-specimen

\begin{tabular}{|c|c|c|c|}
\hline Variables & \multicolumn{3}{|c|}{ Breast size } \\
\hline No. of breasts $(n=174)$ & $51(29.31)$ & $66(37.93)$ & $57(32.57)$ \\
\hline \multicolumn{4}{|l|}{ Mastectomy, mean [min-max] } \\
\hline Weight (kg) & $217.43[55-290]$ & 399.43 [300-490] & $693.89[490-1,220]$ \\
\hline \multicolumn{4}{|l|}{ Patient variables } \\
\hline Age (year), mean \pm SD & $41.01 \pm 8.49$ & $44.88 \pm 10.97$ & $44.60 \pm 44.14$ \\
\hline Weight $(\mathrm{kg})$, mean $\pm \mathrm{SD}$ & $52.18 \pm 5.30$ & $56.44 \pm 5.85$ & $63.89 \pm 8.56$ \\
\hline $\mathrm{BMI}\left(\mathrm{kg} / \mathrm{m}^{2}\right)$, mean $\pm \mathrm{SD}$ & $20.12 \pm 2.25$ & $21.92 \pm 3.30$ & $24.93 \pm 3.42$ \\
\hline Current smoker & $3(5.26)$ & 0 & 0 \\
\hline DM & 0 & $2(3.03)$ & 0 \\
\hline HTN & $2(3.50)$ & $5(7.58)$ & $7(13.73)$ \\
\hline Previous breast operation & $12(23.53)$ & $10(15.15)$ & $4(7.02)$ \\
\hline \multicolumn{4}{|l|}{ Pathology } \\
\hline Invasive ductal carcinoma & $28(49.12)$ & $35(53.03)$ & $29(56.86)$ \\
\hline Ductal carcinoma in situ & $17(29.82)$ & $28(42.42)$ & $14(27.45)$ \\
\hline Invasive lobular carcinoma & $3(5.26)$ & $3(4.55)$ & $4(7.84)$ \\
\hline II & $14(27.45)$ & 26 (39.39) & $22(38.60)$ \\
\hline III & $7(13.72)$ & $10(15.15)$ & $8(14.06)$ \\
\hline \multicolumn{4}{|l|}{ Neoadjuvant/adjuvant therapy } \\
\hline $\begin{array}{l}\text { Neoadjuvant radiation } \\
\text { therapy }\end{array}$ & $5(9.80)$ & $7(10.60)$ & $1(1.75)$ \\
\hline Adjuvant radiation therapy & $9(17.64)$ & $7(10.60)$ & $17(29.82)$ \\
\hline Neoadjuvant chemotherapy & $12(23.52)$ & $13(19.70)$ & $10(17.54)$ \\
\hline Adjuvant chemotherapy & 18 (35.29) & 19 (28.79) & $18(31.58)$ \\
\hline \multicolumn{4}{|l|}{ Breast surgeon } \\
\hline Surgeon 1 & $31(60.78)$ & $40(60.61)$ & 37 (64.91) \\
\hline Surgeon 2 & $14(27.45)$ & $13(19.70)$ & $10(17.54)$ \\
\hline Surgeon 3 & $6(11.76)$ & $13(19.70)$ & $10(17.54)$ \\
\hline
\end{tabular}

Table 1 (continued) 
Table 1 (continued)

\begin{tabular}{lccc}
\hline \multirow{2}{*}{ Variables } & \multicolumn{3}{c}{ Breast size } \\
\cline { 2 - 4 } Type of mastectomy & Small breast, $\mathrm{n}(\%)$ & Medium breast, $\mathrm{n}(\%)$ & Large breast, $\mathrm{n}(\%)$ \\
Skin sparing & $20(39.21)$ & $23(34.85)$ & $20(35.09)$ \\
Nipple sparing & $26(50.98)$ & $40(60.61)$ & $31(54.39)$ \\
Radical (modified) & $5(9.80)$ & $3(4.55)$ & $6(10.53)$ \\
Lymph nose dissection & & & $6(10.53)$ \\
$(-)$ & $7(13.73)$ & $15(22.73)$ & $14(24.56)$ \\
Axillary & $14(27.45)$ & $47(71.21)$ & $37(64.91)$ \\
Sentinel & $30(58.82)$ & & \\
\hline
\end{tabular}

*, other pathologies: mucinous carcinoma, papillary carcinoma, myxoid tumor, Paget's disease. SD, standard deviation; BMI, body mass index; DM, diabetes mellitus; HTN, hypertension.

Table 2 Postoperative complications based on breast size

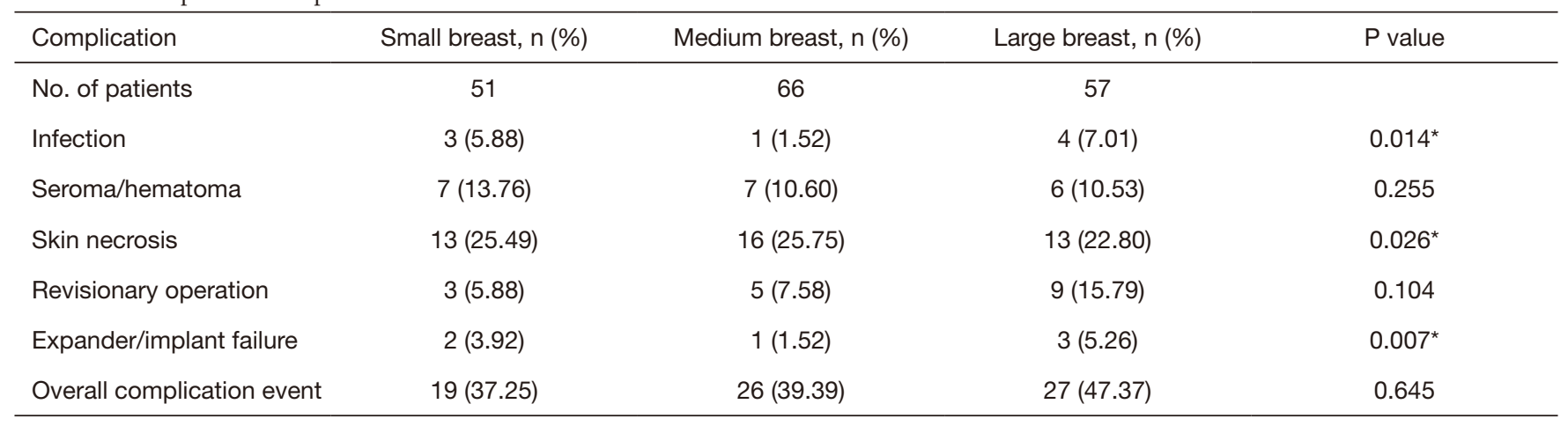

*, statistically significant.

and skin necrosis were also higher in small breasts, although the difference was not significant $(\mathrm{P}=0.255$ and 0.296 , respectively). Revision surgery was more frequently performed in large breasts, although the difference did not reach significance $(\mathrm{P}=0.104)$.

\section{Risk factors of complications}

Univariate logistic regression analysis identified BMI, breast volume, weight, radiation, chemotherapy, type of mastectomy, type of lymph nose dissection, and expander characteristics as significant variables of complications. In multivariate logistic regression analysis, BMI [odds ratio (OR): 1.25 ; $95 \%$ confidence interval (CI): 1.08-1.44; $\mathrm{P}=0.003]$, nipple-sparing mastectomy (OR: 2.82, 95\% CI: 1.07-7.45, $\mathrm{P}=0.036$ ), sentinel lymph node biopsy (OR: 5.10;
95\% CI: 1.36-19.11; $\mathrm{P}=0.016)$, final expansion volume (OR: 0.99; 95\% CI: 0.99-1.00; $\mathrm{P}=0.022$ ), and expansion velocity of the expander (OR: 0.703; 95\% CI: 0.52-0.95; $\mathrm{P}=0.024$ ) were significant independent predictors of any complication (Table 3). Statistically, OR can interpretated as a positive relationship when the value is more than 1 and a negative relationship when less than 1 . Both final expansion volume and velocity showed values less than 1 . Interestingly, a large final expansion volume and rapid expansion velocity of expander negatively influenced the occurrence of overall complications.

Using breast weight, breast volume, and expansion velocity, ROC analysis showed the cut-off value for each complication. Among complications and rate of revision surgery, only the ROC curve for the revision surgery was found to be statistically significant [volume: area under 
Table 3 Multivariate logistic regression analysis of the risk factors of overall complications in two-stage breast reconstruction

\begin{tabular}{|c|c|c|}
\hline Variables & OR $(95 \% \mathrm{Cl})$ & $P$ value \\
\hline BMI & $1.25(1.08-1.44)$ & $0.003^{*}$ \\
\hline Breast volume & $1.01(1.03-0.99)$ & 0.158 \\
\hline Breast weight & $0.98(1.00-0.96)$ & 0.209 \\
\hline Adjuvant radiation therapy & $2.72(0.91-8.15)$ & 0.073 \\
\hline Neoadjuvant chemotherapy & $2.38(0.93-6.10)$ & 0.072 \\
\hline \multicolumn{3}{|l|}{ Type of mastectomy } \\
\hline Skin sparing & 1.00 & 0.072 \\
\hline Nipple sparing & $2.82(1.07-7.45)$ & $0.036^{*}$ \\
\hline Radical (modified) & $0.85(0.17-4.37)$ & 0.850 \\
\hline \multicolumn{3}{|l|}{ Lymph nose dissection } \\
\hline No & 1.000 & 0.053 \\
\hline Axillary & $3.598(0.76-16.87)$ & 0.104 \\
\hline Sentinel & $5.103(1.36-19.11)$ & $0.016^{*}$ \\
\hline \multicolumn{3}{|l|}{ Expander } \\
\hline Final expansion volume & $0.995(0.99-1.00)$ & $0.022^{*}$ \\
\hline Expansion velocity & $0.703(0.52-0.95)$ & $0.024^{*}$ \\
\hline
\end{tabular}

*, statistically significant. OR, odds ratio; $\mathrm{Cl}$, confidence interval; BMI, body mass index.

the curve $(\mathrm{AUC})=0.693, \mathrm{P}=0.017$; weight: $\mathrm{AUC}=0.702$, $\mathrm{P}=0.012]$. Using this cut-off value, breast volume $>505 \mathrm{cc}$ predicted the possibility of revision surgery, with a sensitivity of $64.3 \%$ and a specificity of $66.2 \%$. Breast weight $>695 \mathrm{~g}$ also predict possibility of revision surgery, with a sensitivity of $42.9 \%$ and a specificity of $81.8 \%$ (Figure 4).

\section{Discussion}

Data on the impact of breast size and expansion protocols on the surgical outcomes of breast reconstruction are scarce. In this study, a large breast size was associated with significantly higher rates of infection and prosthetic failure, although skin necrosis was more frequent in medium breasts. In addition, the final expansion volume and expansion velocity were negatively associated with the occurrence of overall complications.

Regarding infection, there are reports that a weight of mastectomy-specimen greater than $800 \mathrm{~g}$ is associated with a higher rate of complications, including ischemia

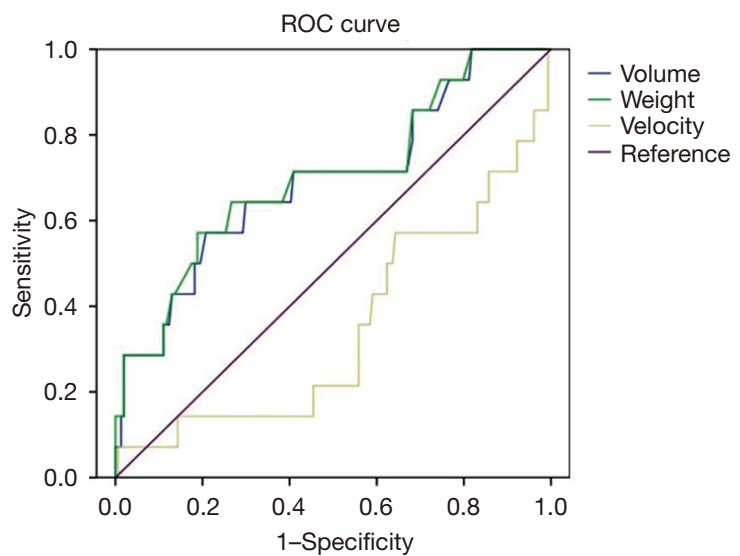

Figure 4 ROC curves for the influencing factors of revision surgery with the breast volume and weight and expansion velocity. A breast volume of $505 \mathrm{cc}$ had an AUC of 0.693; sensitivity, 0.643; and specificity, 0.662. A breast weight of $695 \mathrm{~g}$ had an AUC of 0.702; sensitivity, 0.429; and specificity, 0.818. ROC, receiver operating characteristic; AUC, area under the curve.

and infection (14). A breast size larger than C cup is also significantly associated with postoperative infection (15). Regarding prosthetic failure, an ambiguous understanding of the effect of large breast size on postoperative complications can lead to inaccurate outcome predictions. However, in this study we demonstrated that among Asian women a large breast weight of mastectomy-specimen $(>500 \mathrm{~g})$ increased the risk of implant failure. Implant failure is directly related to implant infection. Increased infection rate in large breasts explains the high failure rate in such cases. However, skin necrosis is higher in medium breasts than in large breasts. In nipple-sparing mastectomy, perfusion to the nipple-areola complex and mastectomy flap is achieved through vasculature in the subdermal and subcutaneous tissues (16). A large breast size results in a large surface area for the mastectomy flap, thus providing low perfusion and leading to increased ischemic sequelae (17). However, for medium-sized breasts, the contact surface between the mastectomy skin flap and implant is smaller than in large breasts, increasing the probability of volume tension on the skin flap during the expansion period. Although this gap has a positive effect on reducing seroma or hematoma through compression, it also has a negative effect on the occurrence of skin necrosis.

High BMI, diabetic smoking, chemotherapy/radiation therapy, and planes of prosthetic insertion or the use of an acellular dermal matrix for implantation are well-known 
risk factors of postoperative complications (18). Similarly, we found that high BMI, nipple-sparing mastectomy, and sentinel lymph node biopsy were independent risk factors. Patients with high BMI have poorer outcomes after breast reconstructive surgery than patients with normal BMI. A multicenter prospective study showed higher risks for overall and major complications in both implant-based and autologous breast reconstruction among obese patients than among underweight/normal-weight patients (8). When obese patients use their upper body, the shearing force caused by the repeated use of the pectoralis muscle is more powerful than in non-obese patients in the immediate postoperative period, which could result in seroma formation in cases of subpectoral placement (8).

The effect of the expansion protocol on the postoperative period has been trivialized. However, interestingly, we found that a large final expansion volume and rapid expansion velocity help lower the occurrence of overall complications, highlighting that the expansion protocol has important effects on the surgical outcomes of two-stage breast reconstruction. Compared with the final expansion volume, the expansion velocity had a more protective effect on the occurrence of overall complications.

Faster expansion can more quickly reduce the dead space and the interval to second surgery; thus, it could shorten the time of biofilm-exposure. Seromas following prosthetic breast reconstruction are complicated by the hypovascularity, pro-inflammatory milieu of the mastectomy skin flap, geometrically complex dead space, and presence of a foreign body with potential contamination and biofilm (19). These factors contribute to the progression of seroma to infection and prosthesis loss (20). Thus, reducing the chance of biofilm formation is strongly associated with reducing seroma and infection.

The effect of breast size should also be considered. Volume expansion may affect the tension on the skin flap, although it can also reduce seroma or hematoma through compression. Meanwhile, expansion velocity allows easier stretching of the skin flap, and a faster expansion velocity shortens the interval between the first and second surgeries. We found that a breast weight $>696 \mathrm{~kg}$ and breast volume $>505 \mathrm{cc}$ increase the probability of revision surgery in the postoperative period. Thus, patients with these characteristics should be closely monitored.

The limitations of this study include its retrospective nature and the categorization of breast size according to the characteristics of Asian women. From the point of view of racial difference, breast size and expectation volume of Asian women are very different from Western women. Expansion velocity and final inflation goal can be affected by these racial factors. Although in our study we controlled possible variables, the results may have limited generalizability in women from Western countries. Especially regarding breast size, Western and Asian women have stark differences. Final implant size and corresponding expander size choice are also affected by the patient's expectation. This may also, in turn, affect the physician's expansion strategy. Furthermore, outcomes such as mastectomy flap necrosis were defined by intervention and thus influenced by surgeon preference. Notably, each complication also carries a different impact based on the reconstruction modality used. For example, mastectomy flap necrosis in implant-based nipple-sparing mastectomy has a higher risk of reconstructive failure than autologous reconstruction. However, the follow-up time was similar to that in other studies examining the outcomes of nipple-sparing mastectomy. Therefore, future research requires the following, a large group study in Asian patient group will be more meaningful, and long-term followup analysis will be needed to understand the effect on the capsular contracture and the resulting operation rate. Additional limitation of this study is means of measurement used to categorize the patients' breast size. Intraoperatively we measured both the mastectomy specimen volume and weight. However, we divided and analyzed patient groups according to weight of mastectomy specimen, because of the error occurred between readers. Lastly, the retrospective nature of this study is a limitation. To provide more specific guidelines for the expansion protocol, we should undertake a prospective study. We hope that we can provide better solutions to breast reconstructive surgeons through future studies.

In conclusion, the final expansion volume and expansion velocity have a significant negative relationship with overall complications in breast reconstruction surgery. However, breast size can be used to predict the possibility of complications. A standard expansion protocol needs to be established to ensure a successful two-stage breast reconstruction.

\section{Acknowledgments}

Funding: This research was supported by a grant of the Korea Health Technology R\&D Project through the Korea Health Industry Development Institute (KHIDI), funded by the Ministry of Health \& Welfare, Republic of Korea (grant number: HI20C2140). 


\section{Footnote}

Reporting Checklist: The authors have completed the STARD reporting checklist. Available at https://gs.amegroups.com/ article/view/10.21037/gs-21-515/rc

Data Sharing Statement: Available at https://gs.amegroups. com/article/view/10.21037/gs-21-515/dss

Peer Review File: Available at https://gs.amegroups.com/ article/view/10.21037/gs-21-515/prf

Conflicts of Interest: All authors have completed the ICMJE uniform disclosure form (available at https://gs.amegroups. com/article/view/10.21037/gs-21-515/coif). All authors report this research was supported by a grant of the Korea Health Technology R\&D Project through the Korea Health Industry Development Institute (KHIDI), funded by the Ministry of Health \& Welfare, Republic of Korea (grant number: HI20C2140). The authors have no other conflicts of interest to declare.

Ethical Statement: The authors are accountable for all aspects of the work in ensuring that questions related to the accuracy or integrity of any part of the work are appropriately investigated and resolved. The study was conducted in accordance with the Declaration of Helsinki (as revised in 2013) and was approved by the Institutional Review Board of Ajou University Hospital (approval number: MED-MDB-21-235). Individual consent for this retrospective analysis was waived.

Open Access Statement: This is an Open Access article distributed in accordance with the Creative Commons Attribution-NonCommercial-NoDerivs 4.0 International License (CC BY-NC-ND 4.0), which permits the noncommercial replication and distribution of the article with the strict proviso that no changes or edits are made and the original work is properly cited (including links to both the formal publication through the relevant DOI and the license). See: https://creativecommons.org/licenses/by-nc-nd/4.0/.

\section{References}

1. Krueger EA, Wilkins EG, Strawderman M, et al. Complications and patient satisfaction following expander/implant breast reconstruction with and without radiotherapy. Int J Radiat Oncol Biol Phys
2001;49:713-21.

2. Schuster RH, Rotter S, Boonn W, et al. The use of tissue expanders in immediate breast reconstruction following mastectomy for cancer. Br J Plast Surg 1990;43:413-8.

3. Ragaz J, Jackson SM, Le N, et al. Adjuvant radiotherapy and chemotherapy in node-positive premenopausal women with breast cancer. N Engl J Med 1997;337:956-62.

4. Berry T, Brooks S, Sydow N, et al. Complication rates of radiation on tissue expander and autologous tissue breast reconstruction. Ann Surg Oncol 2010;17 Suppl 3:202-10.

5. Delanian S, Lefaix JL. The radiation-induced fibroatrophic process: therapeutic perspective via the antioxidant pathway. Radiother Oncol 2004;73:119-31.

6. Gurunluoglu R, Gurunluoglu A, Williams SA, et al. Current trends in breast reconstruction: survey of American Society of Plastic Surgeons 2010. Ann Plast Surg 2013;70:103-10.

7. Lin KY, Blechman AB, Brenin DR. Implant-based, two-stage breast reconstruction in the setting of radiation injury: an outcome study. Plast Reconstr Surg 2012;129:817-23.

8. Srinivasa DR, Clemens MW, Qi J, et al. Obesity and Breast Reconstruction: Complications and PatientReported Outcomes in a Multicenter, Prospective Study. Plast Reconstr Surg 2020;145:481e-90e.

9. McCue JD, Migliori M, Cunningham BL. Expanders and breast reconstruction with gel and saline implants. In: Hall-Findlay E, Evans G. editors. Aesthetic and Reconstructive Surgery of the Breast. Philadelphia: Saunders, 2010:29-50.

10. Yang JD, Lee JW, Cho YK, et al. Surgical techniques for personalized oncoplastic surgery in breast cancer patients with small- to moderate-sized breasts (part 1): volume displacement. J Breast Cancer 2012;15:1-6.

11. Duggal CS, Grudziak J, Metcalfe DB, et al. The effects of breast size in unilateral postmastectomy breast reconstruction. Ann Plast Surg 2013;70:506-12.

12. Yang JD, Lee JW, Cho YK, et al. Surgical techniques for personalized oncoplastic surgery in breast cancer patients with small- to moderate-sized breasts (part 2): volume replacement. J Breast Cancer 2012;15:7-14.

13. Khansa I, Hendrick RG Jr, Shore A, et al. Breast reconstruction with tissue expanders: implementation of a standardized best-practices protocol to reduce infection rates. Plast Reconstr Surg 2014;134:11-8.

14. Frey JD, Salibian AA, Karp NS, et al. The Impact of Mastectomy Weight on Reconstructive Trends and Outcomes in Nipple-Sparing Mastectomy: Progressively 
Greater Complications with Larger Breast Size. Plast Reconstr Surg 2018;141:795e-804e.

15. Francis SH, Ruberg RL, Stevenson KB, et al. Independent risk factors for infection in tissue expander breast reconstruction. Plast Reconstr Surg 2009;124:1790-6.

16. Seitz IA, Nixon AT, Friedewald SM, et al. "NACsomes": A new classification system of the blood supply to the nipple areola complex (NAC) based on diagnostic breast MRI exams. J Plast Reconstr Aesthet Surg 2015;68:792-9.

17. Gdalevitch P, Ho A, Genoway K, et al. Direct-to-implant single-stage immediate breast reconstruction with acellular dermal matrix: predictors of failure. Plast Reconstr Surg 2014;133:738e-47e.

Cite this article as: Kim MJ, Lee WB, Lee IJ, Hahn HM, Thai DQ, Kim JY. The investigation of the relation between expansion strategy and outcomes of two-stage expander-implant breast reconstruction. Gland Surg 2022;11(1):1-11. doi: 10.21037/gs-21-515
18. Endara M, Chen D, Verma K, et al. Breast reconstruction following nipple-sparing mastectomy: a systematic review of the literature with pooled analysis. Plast Reconstr Surg 2013;132:1043-54.

19. Jordan SW, Khavanin N, Kim JYS. Seroma in Prosthetic Breast Reconstruction. Plast Reconstr Surg 2016;137:1104-16.

20. Woerdeman LA, Hage JJ, Smeulders MJ, et al. Skinsparing mastectomy and immediate breast reconstruction by use of implants: an assessment of risk factors for complications and cancer control in 120 patients. Plast Reconstr Surg 2006;118:321-30; discussion 331-2. 